\title{
Role of Air in Granular Jet Formation
}

\author{
Gabriel Caballero, Raymond Bergmann, Devaraj van der Meer, Andrea Prosperetti, and Detlef Lohse \\ Faculty of Science and J. M. Burgers Centre for Fluid Dynamics, University of Twente, 7500 AE Enschede, The Netherlands
}

(Received 19 January 2007; published 6 July 2007)

A steel ball impacting on a bed of very loose, fine sand results in a surprisingly vigorous jet which shoots up from the surface of the sand [D. Lohse et al., Phys. Rev. Lett. 93, 198003 (2004)]. When the ambient pressure $p$ is reduced, the jet is found to be less vigorous [R. Royer et al., Nature Phys. 1, 164 (2005)]. In this Letter we show that $p$ also affects the rate of penetration of the ball: Higher pressure increases the rate of penetration, which makes the cavity created by the ball close deeper into the sand bed, where the hydrostatic pressure is stronger, thereby producing a more energetic collapse and jetting. The origin of the deeper penetration under normal ambient pressure is found to lie in the extra sand fluidization caused by the air flow induced by the falling ball.

DOI: 10.1103/PhysRevLett.99.018001

When an object impacts on a bed of fine, loose grains, it is quickly engulfed and a surprisingly vigorous jet shoots upward from the surface of the sand [1-4], similar to what happens in a liquid [5-7]. Royer et al. [4] found a granular jet created at reduced ambient pressure to be smaller than at atmospheric pressure, highlighting the relevant role that interstitial air plays in systems with very small grains $(<100 \mu \mathrm{m})[8-10]$.

In this Letter we present experiments where we found that the pressure-dependent reduction of the jet height goes hand in hand with a reduction of the final depth reached by the ball. We propose that it is this change in the penetration rate which alters the size of the jet. Moreover, we show that the reduction of the jet height with pressure is consistent with a mechanism of jet formation governed by the gravitational collapse of the void as proposed in [2]. Finally, we discuss experiments which suggest that the flow of air around the moving ball is responsible for the influence that the ambient pressure has on the drag force the ball experiences inside the sand.

For the experiments presented here we adapted the setup used in $[2,3]$ to allow for the evacuation of air. It consists of a deep bed $(\approx 40 \mathrm{~cm})$ of sand grains between 20 and $60 \mu \mathrm{m}$ in size and with shapes of equivalent eccentricities between 0.2 and 0.6 . The density of the material composing the grains is $2.21 \pm 0.04 \mathrm{~g} / \mathrm{cm}^{3}$, its angle of repose is about $26^{\circ}$, and the volume fraction obtained by pouring it into a container is around $46 \%$. The cross section of the granular bed is a square of $14 \mathrm{~cm}$ on a side. Before each impact experiment, air was blown through the bed from the bottom in order to decompactify and homogenize the sand. Then the air supply was slowly reduced to zero and the bed was allowed to gently settle into a static, loose $(41 \%$ volume fraction), weakened state. The container was subsequently sealed off and the air was slowly pumped out simultaneously from above and below the bed, with the pressure falling at a rate of 5 mbar per second. We verified that there was no difference in measurements done when the evacuation of air was done at slower rates. When the desired pressure was attained, the valves were closed and a
PACS numbers: 45.70.-n, 47.55.Lm, 47.57.Gc

steel ball of diameter $D=1.6 \mathrm{~cm}$ and mass $m=16.5 \mathrm{~g}$ was released from different heights. Note that, given the small size of the grains, cohesive forces are non-negligible and may play a role in the behavior of the granular material [11].

A high-speed camera filming from one side through the transparent walls of the container allowed us to measure the maximum height $h$ reached by the tip of the jet. The complete trajectory $z(t)$ of the ball inside the sand, from the moment of impact until its final depth $z_{\text {final }}$, was measured by attaching to it a thin thread with markers, which was kept tight during the process by running it through a small fastener. Because of the friction in this fastener, the measured effective acceleration of the falling ball before impact was about $10 \%$ smaller than gravity. In the following, each experimental point plotted is the average of three independent realizations, and the error bar is the corresponding standard deviation.

The maximum height of the jet $h$ and the final penetration depth of the ball $z_{\text {final }}$ are plotted as a function of the ambient pressure $p$ in Figs. 1(a) and 1(b). In these plots four different impact velocities $v_{0}$ were used, which are expressed in terms of the Froude number, defined as $\mathrm{Fr} \equiv$ $2 v_{0}^{2} / g D$, where $g$ is the acceleration of gravity. As expected [4], $h$ first increases with increasing ambient pressure. But also $z_{\text {final }}$ increases, revealing a remarkable strong dependence on pressure. For pressures higher than $p \approx$ 400 mbar the jet height saturates, unlike the penetration depth which has a more monotonic behavior. By plotting the jet height versus the final depth [Fig. 1(c)] two distinct regimes are revealed: (i) for low pressures, $h$ increases more or less linearly with $z_{\text {final }}$ (dashed line), and (ii) for higher $p$ the jet height saturates to a (Froude-dependent) constant value, whereas the penetration depth continues to increase.

In order to explain the above observations we now compare, for a single impact velocity, the complete trajectories $z(t)$ of the ball at four different pressures, depicted in Fig. 2(a). The vertical dotted line marks the closure time $t_{c}$ of the cavity at $p=400$ mbar, which we were able to 


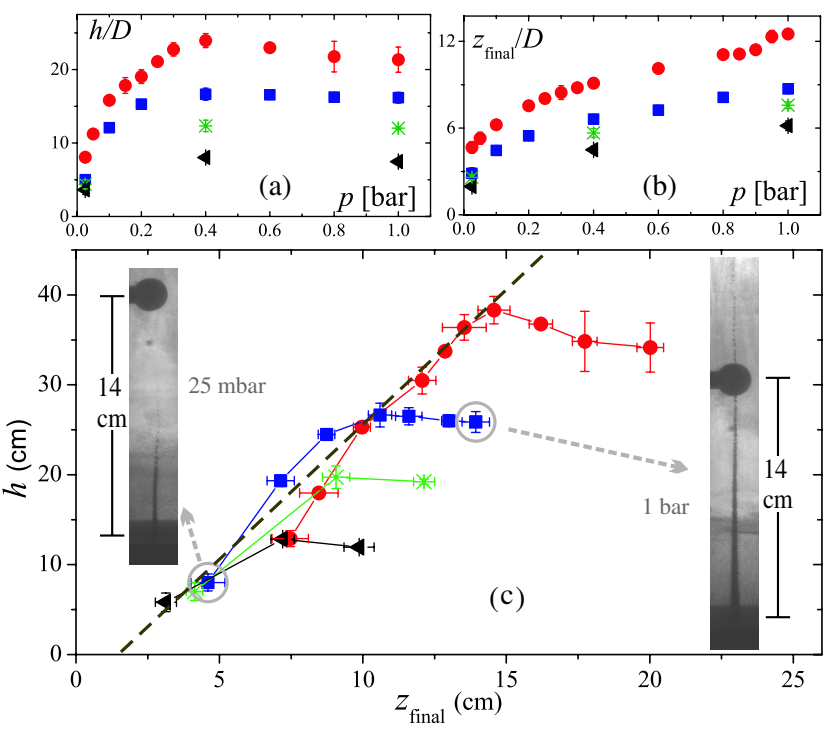

FIG. 1 (color online). (a) Maximum height of the jet $h$, and (b) final depth of the ball $z_{\text {final }}$, as a function of the ambient pressure $p$ for different Froude numbers: $\mathrm{Fr}=7$ ( 4$), 18(\star)$, 35 (ם), and $132(\mathbf{a}) . D=1.6 \mathrm{~cm}$ is the diameter of the impacting ball. (c) Maximum height $h$ of the jet versus the penetration depth $z_{\text {final }}$ of the ball. The lines in this plot are guides to the eye.

measure by a top-view, high-speed recording of the impact and cavity collapse [12]. The tangents of the trajectories at the moment of impact $(t=0)$ are identical, but for $t>0$ they start to diverge. A suitable measure for this divergence is the location $z_{i}$ of the ball at $t=t_{c}$, which can be determined from the trajectories [Fig. 2(b)]. Clearly, for higher pressures ( $p \gtrsim 400 \mathrm{mbar}$ ) the values of $z_{i}$ differ very little, reflecting the fact that trajectories are almost identical up to the time when the cavity closes. Therefore we infer that the cavity dynamics, the closure depth, and consequently also the jet formation process are the same. This explains why the jet height becomes constant for higher pressures: The impact velocity $v_{0}$ determines the jet height in this first regime.

On the other hand, at low pressures $(p<400$ mbar) the trajectories deviate substantially during the interval $0 \leq$ $t \leq t_{c}$. Therefore, eventually the penetration rate (and, with it, $z_{\text {final }}$ ) becomes the determining factor for the closure depth and consequently also for the jet height $h$. So, in this second regime, $h$ will become independent of the impact velocity and will correlate with $z_{\text {final }}$, completely in agreement with our observations in Fig. 1(c).

To describe the creation and subsequent collapse of the impact cavity we combine the drag force model for the impacting ball of [3] and the collapse model introduced in [2]. The drag force model of [3] is based on a drag force of the Coulomb form $F_{d}=-\kappa z$, where $z$ is the depth of the ball and $\kappa$ is a constant. This leads to the equation of motion $m \ddot{z}=m g-\kappa z$, with initial conditions $z(0)=0$ and $\dot{z}(0)=v_{0}$, which is readily solved to obtain the trajectory of the ball in the sand
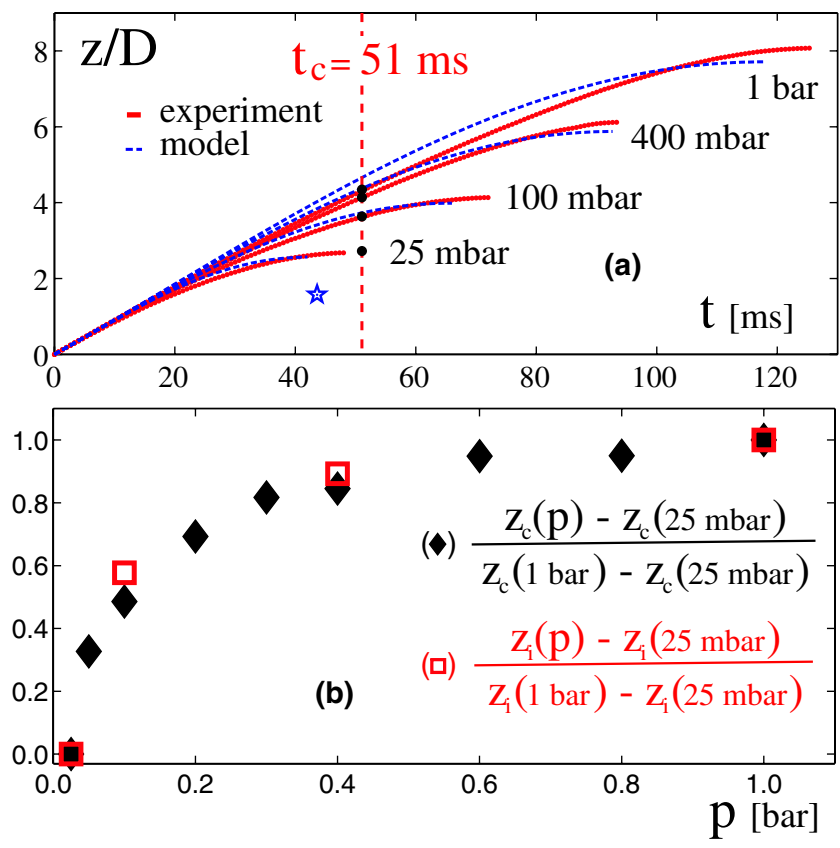

FIG. 2 (color online). (a) Experimental trajectories of the ball in the sand (thick lines) for $\mathrm{Fr}=35$ compared to the drag force model, Eq. (1) (dashed lines), with $\kappa$ the only fitting parameter. The vertical dotted line indicates the measured closure time $t_{c}$ of the cavity. The black dots mark the location $z_{i}$ of the ball at closure. The star indicates the calculated closure time $t_{c}$ and closure depth $z_{c}$ using the model [Eqs. (1) and (2)]. (b) Location of the ball at closure $z_{i}$ (squares) obtained from the top figure, and the closure depth $z_{c}$ (diamonds) predicted by the model, both as a function of pressure. In order to show that the two quantities follow the same trend, we plotted them shifted and normalized such that they go from 0 at 25 mbar to 1 at 1 bar, with $z_{c}(25 \mathrm{mbar})=1.43 D, \quad z_{c}(1 \mathrm{bar})=1.57 D, \quad z_{i}(25 \mathrm{mbar})=$ $2.68 D$, and $z_{i}(1 \mathrm{bar})=4.33 D$.

$$
z(t)=\frac{g m}{\kappa}\left[\sqrt{\frac{\operatorname{Fr} \bar{D} \kappa}{2 g m}} \sin \left(\sqrt{\frac{\kappa}{m}} t\right)-\cos \left(\sqrt{\frac{\kappa}{m}} t\right)+1\right],
$$

for $0 \leq t \leq t_{\text {stop }}$, where $t_{\text {stop }}$ is defined by the condition $\dot{z}\left(t_{\text {stop }}\right)=0$. Inserting $t_{\text {stop }}$ into the above equation gives the final depth the ball reaches as $z_{\text {final }}=(\mathrm{gm} / \kappa)[1+(1+$ $\kappa D \mathrm{Fr} / 2 \mathrm{gm})^{1 / 2}$. In [3] it was shown that this model leads to an accurate description of the trajectory of the ball in our loose, fine sand at atmospheric pressure and zero impact velocity. To evaluate how the model performs for $v_{0}>0$ and at lower ambient pressures, in Fig. 3(a) we compare the measured $z_{\text {final }}$ as a function of the Froude number to the prediction of the model at three different pressures. The solid lines in Fig. 3(a) are the best fits of the equation for $z_{\text {final }}$ to the experimental data, where the only free parameter is the drag force coefficient $\kappa$. The agreement between the model and the experiments is very good for low and intermediate Froude numbers, but differences arise when $\mathrm{Fr} \gtrsim 80$. A plausible explanation of such behavior is that some velocity-dependent term needs to be included in the drag force on the ball (see, e.g., [11,13-16]), whose rela- 
(a)

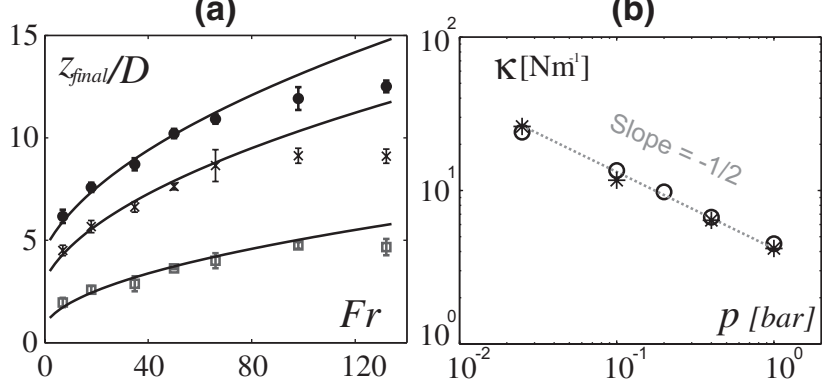

FIG. 3. (a) Final depth as a function of the Froude number at different pressures $p=1(\bigcirc), 0.4(\star)$, and 0.025 bar $(\square)$. The lines are fits using the prediction of $z_{\text {final }}$ by the force model, where the drag force coefficient $\kappa$ is the only free parameter. The values of $\kappa$ that result from the fits are plotted as circles in (b), suggesting a relation of the form $\kappa \propto p^{-1 / 2}$. The stars in (b) result from the fitting of Eq. (1) to the experimental trajectories in Fig. 2(a) (dashed lines).

tive weight would increase with Fr. From the fitting procedure leading to Fig. 3(a) we obtain the dependence of $\kappa$ on the ambient pressure plotted in Fig. 3(b) (circles), which suggests a power-law relation of the form $\kappa \propto p^{-1 / 2}$. The stars in Fig. 3(b) result from the fitting of Eq. (1) to the experimental trajectories in Fig. 2(a) (dashed lines). The agreement between the experimental and the computed trajectories is reasonably fair, but not as good as it was at zero impact velocity [3]. This could be improved by supplementing the model with a velocity-dependent term which would be relatively more important at higher Froude numbers.

Now that we know the trajectory of the ball in the sand at different pressures, we can estimate the time $t_{c}$ and depth $z_{c}$ at which the void created by the ball first closes during its collapse, by proceeding in the same way as in [2]: The cavity is assumed to be cylindrical upon creation and starts to collapse radially immediately after the ball passes. Thus, the total time needed for the cavity to close at depth $z$ is found by adding the time $t_{\text {pass }}(z)$ the ball needs to reach this depth [which follows directly from Eq. (1)] and the time $t_{\text {coll }}(z)$ needed for the subsequent collapse at depth $z$. This last quantity follows from the two-dimensional Rayleigh equation introduced in [2], in which, at each depth $z$, the pressure difference causing the collapse is taken proportional to $g z$. This equation can conveniently be written in nondimensional form by introducing $\hat{R}=2 R / D$ and $\hat{t}=$ $2 t \sqrt{g z} / D$,

$$
\left(\hat{R} \ddot{\hat{R}}+\dot{\hat{R}}^{2}\right) \log \frac{\hat{R}}{\hat{R}_{\infty}}+\frac{1}{2} \dot{\hat{R}}^{2}=1,
$$

with initial conditions $\hat{R}(0)=1$ and $\dot{\hat{R}}(0)=0$.

By solving Eq. (2) we find a unique solution $\hat{R}(\hat{t})$, which becomes zero at $\hat{t}=\hat{t}_{\text {coll }}$, and which only depends on the value of $\hat{R}_{\infty}$. The $z$ dependence of the dimensional collapse time $t_{\text {coll }}$ now follows from $t_{\text {coll }}=\hat{t}_{\text {coll }} D /(2 \sqrt{g z})$. If we equate $R_{\infty}$ with half the container size [7], we find $\hat{t}_{\text {coll }}=$
0.78. The depth $z_{c}$ and time $t_{c}$ at which the cavity will collapse is now found by determining the minimum of this total time $t_{\text {total }}(z)=t_{\text {pass }}(z)+t_{\text {coll }}(z)$ with respect to $z$. The resulting closure time $t_{c}$ turns out to hardly depend on pressure, and is plotted as a single point (star) in Fig. 2(a). The calculated closure depth $z_{c}$, however, does depend on $p$, and is compared to the experimentally determined location $z_{i}$ of the ball at closure time in Fig. 2(b). Note that $z_{i}$ and $z_{c}$ show a remarkably similar trend: Both are more or less constant for high pressures and show a sharp decrease below $p \approx 400$ mbar.

The potential energy of the jet at its maximum height, proportional to $h$, arises from the kinetic energy of the sand as it flows inward to fill up the void at a depth $z_{c}$ below the surface. This kinetic energy is due to the potential energy stored in the void in the neighborhood of the depth $z_{c}$ after the passage of the ball, which is in turn proportional to $z_{c}$ itself. This is because this depth determines the hydrostatic pressure difference causing the collapse of the void as noted before in connection with (2). If, on the basis of experimental observation, the jet diameter is at most only weakly dependent on the closure depth, we conclude that $h \propto z_{c}$, thus explaining not only the fact that $h$ and $z_{c}$ follow the same trend but also the observation of Fig. 1(c) that, at low pressure (approximately $<400$ mbar), when $z_{\text {final }}$ and $z_{c}$ are closely correlated [see Fig. 2(a)], we find a linear relation between $z_{\text {final }}$ and $h$. At higher pressures $z_{\text {final }}$ and $z_{c}$ are no longer correlated, and also $h$ becomes independent of $z_{\text {final }}$.

We conclude that the formation of the jet is solely due to the violent gravitational collapse of the cavity that is created upon impact, and that the ambient air plays only an indirect role in this process by modifying the trajectory of the ball in the sand. This is in contradiction with the mechanism proposed by Royer et al. [4], where the jet would be driven by a pressurized air pocket trapped in the sand. They arrived at this conclusion by studying a thickthin jet structure in a setup similar to ours but using higher impact velocities and a bigger ball $(2.2 \mathrm{~cm}$ in diameter), and combining these measurements with $\mathrm{x}$-ray imaging of jets formed in a sand bed inside a much smaller container (a $12 \mathrm{~mm}$ ball dropped into a 75-mm-deep bed in a cylinder with inner diameter $30 \mathrm{~mm}$ ). We did not observe the thick jet with the $1.6 \mathrm{~cm}$ ball at low impact velocities, but we did observe it for a $2.5 \mathrm{~cm}$ ball. This makes us think that the thick jet structure is due to boundary effects, but this phenomenon needs to be investigated further. Besides, our measured closure times, closure depths, and trajectories show that at high ambient pressures the ball is still moving downwards at considerable speed at the moment of cavity closure and formation of the jet, which is not considered in the model by Royer et al. We think that the features of the cavity dynamics observed with their x-ray setup are specific to the small container size used for these measurements.

But how does the ambient pressure modify the rheological properties of the sand? We believe that the flow of 


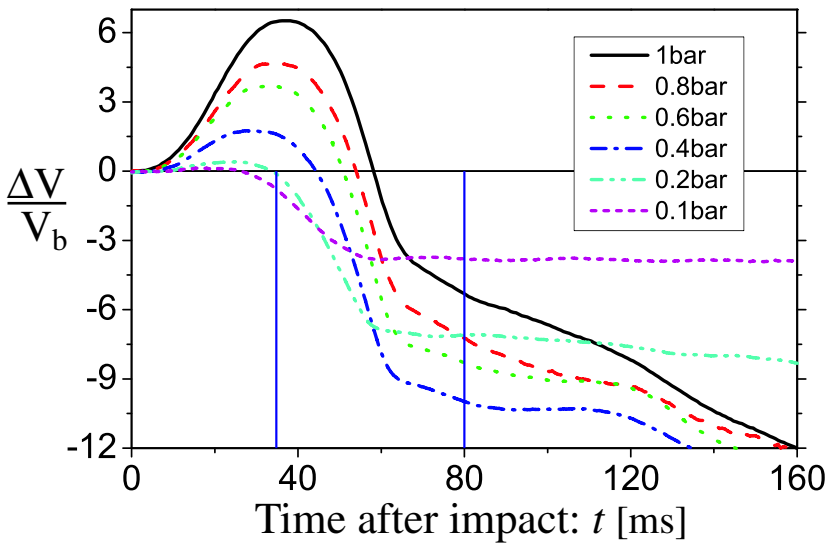

FIG. 4 (color online). $\Delta V=V(t)-V(t=0)$ for $\mathrm{Fr}=132$, where $V(t)$ is the total volume occupied by the sand bed and $t=$ 0 is the moment of impact. $V_{b}$ is the volume of the impacting ball with $D=1.6 \mathrm{~cm}$. Each curve is the average of three independent experiments. The vertical lines show, in chronological order and for 0.4 bar, the closure time obtained with the model, and the first time at which the jet can be seen $(\approx 5 \mathrm{~cm}$ above the surface). The jet tip reaches its maximum height at $\approx 330 \mathrm{~ms}$.

interstitial air that the ball creates while moving through the sand further fluidizes the sand, effectively reducing the drag on the ball. When the pressure is reduced there is less interstitial air, and the fluidization would be less important. In order to test this idea we studied the change in the total volume occupied by the sand bed after the impact of the ball by imaging with a fast video camera at 1500 frames per second a small region (of the order of $D$ ) at the center of one of the lateral, transparent walls of the container at the level of the bed surface. As the surface is found to rise evenly in the container, the product of the cross section of the container and the total height of the bed measured with the camera gives us an estimate of the total volume $V(t)$ occupied by the sand bed at time $t$. Figure 4 shows $\Delta V=$ $V(t)-V(t=0)$ for $\mathrm{Fr}=132$ and at different pressures, where $t=0$ is the moment of impact. It can be seen that the bed expands more at higher pressure, which is in accordance with our hypothesis of the fluidization of the bed due to the flow of interstitial air.

Moreover, if one compares the (lower Fr) trajectories of Fig. 2(a) with the time scale of the rising in Fig. 4, one finds that, at least for pressures above 400 mbar, the trajectories are almost identical while the expansion of the bed in this pressure range shows large differences. It can therefore be excluded that the observed differences in bed height can be attributed solely to variations in the volume of the cavity of different trajectories, and the expansion of the bed must therefore be related to the levitation of sand by the interstitial air flow.

The fact that the bed rises evenly over the container does not discard our local fluidization argument: In spite of the above, the main cause for the height increase is that sand needs to be displaced for the creation of the cavity. It merely means that in the unlimited case the area which would be affected would be larger than the cross section of our present container. One then may also wonder about the influence of the boundaries on the cavity collapse dynamics. However, the fact that identical trajectories up to the closure time at different pressures lead to jets of the same maximum height, and at the same time the associated expansion of the bed can be very different, suggests that the boundaries do not play an important role on the cavity collapse.

In conclusion, we found that ambient air does not play a direct role in the formation of a granular jet in loose, fine sand. Instead, we have shown that the jet is less vigorous at reduced pressures because the ball penetrates less deep into the sand and consequently the collapse of the void is less violent. The effect of the ambient pressure on the intensity of the jet is indeed closely related to the "dynamic coupling between gas and granulate motion" [4]. Our experiments show that the origin thereof is that also the trajectory of the ball is strongly influenced by the ambient pressure, presumably because the air flow around the moving ball partially levitates the grains and effectively reduces the drag force on the ball. The challenge remains to obtain direct experimental evidence and a quantitative model to support and improve our understanding of this phenomenon.

We would like to thank Martin Bos, Gert-Wim Bruggert, and Jan Talman for advice on and construction of the experimental setup. The work is part of the research program of FOM, which is financially supported by NWO; G.C. and R. B. acknowledge financial support.

[1] S. T. Thoroddsen and A. Q. Shen, Phys. Fluids 13, 4 (2001).

[2] D. Lohse et al., Phys. Rev. Lett. 93, 198003 (2004).

[3] D. Lohse et al., Nature (London) 432, 689 (2004).

[4] J. R. Royer et al., Nature Phys. 1, 164 (2005).

[5] A. Prosperetti and H. N. Oguz, Annu. Rev. Fluid Mech. 25, 577 (1993).

[6] J.E. Hogrefe et al., Physica (Amsterdam) 123D, 183 (1998).

[7] R. Bergmann et al., Phys. Rev. Lett. 96, 154505 (2006).

[8] H. K. Pak, E. Van Doorn, and R. P. Behringer, Phys. Rev. Lett. 74, 4643 (1995).

[9] X. Yan et al., Phys. Rev. Lett. 91, 014302 (2003).

[10] M.E. Möbius et al., Phys. Rev. Lett. 93, 198001 (2004).

[11] A. M. Walsh et al., Phys. Rev. Lett. 91, 104301 (2003).

[12] See EPAPS Document No. E-PRLTAO-99-027728 for a movie.mpg: A top-view, high-speed recording of the impact and the cavity collapse. $\mathrm{Fr}=25, p=200 \mathrm{mbar}$, $D=1.6 \mathrm{~cm}$. For more information on EPAPS, see http://www.aip.org/pubservs/epaps.html.

[13] J. S. Uehara et al., Phys. Rev. Lett. 90, 194301 (2003).

[14] M. B. Stone et al., Nature (London) 427, 503 (2004).

[15] M. P. Ciamarra et al., Phys. Rev. Lett. 92, 194301 (2004).

[16] L. S. Tsimring and D. Volfson, in Powders and Grains 2005, edited by R. Garcia-Rojo, H. J. Herrmann, and S. McNamara (Taylor and Francis, London, 2005), p. 1215. 